\title{
Analyzing the Impact of Micro Mobility in Wireless Adhoc Networks
}

\author{
Moazzam Jawaid, Mukhtiar Ali Unar, and Naveed Ahmed Jaffari
}

\begin{abstract}
An adhoc network can be visualized as collection of wireless devices, dynamically creating a short lived network lacking centralized infrastructure where all nodes rely on each other for providing route information in the network. The highly dynamic nature of the nodes needs a very robust and effective routing algorithm. Recently several routing protocols have been projected but few have been implemented yet and prominent among them are DSDV, AODV, TORA, and DSR. It is of extreme importance to deploy a suitable routing protocol as the shift in the routing phenomena can lead to reckonable amount of divergence in efficiency of the networks. In this study, the impact of the mobility has been analyzed on the network efficiency by simulating different scenarios of wireless adhoc networks in NS-2. We have studied the network performance in terms of associated control traffic, packet delivery ratio, and average delay between source $\&$ destination. These results can be used as standard showing the suitability of different routing protocols in specific condition as well as in future these can be used to integrate the security phenomena in AODV.
\end{abstract}

\section{Index Terms-AODV, DSR, MANET, TCP, NS2.}

\section{INTRODUCTION}

Networking is the process of interconnecting computing devices together in order to take the optimal advantage of the available resources by allowing electronic communication among those nodes. If a company or user wishes to make data mobile, portable and accessible anywhere then wireless networking is the solution. A wireless networking system helps user to reduce the possible downtime one would normally have in a wired environment due to cable related problems. Wireless networking has emerged as a substitute that allows people to communicate irrespective of their geographic locations. Two different operating modes of wireless network include.

- Infrastructure based Networks

- Infrastructure-less (Adhoc) Networks

\section{A. Infrastructure Based Networks}

The term infra structured refers to a network with preset gateways. A mobile device contacts a predetermined base station within its range and base station helps mobile node to establish communication with the intended destinations. As node moves out of the range of a particular base station, it searches \& establishes connection starts communicating through it. Change of access point in this way is called handoff and it may arise problem in smooth communication.

Manuscript received December 30, 2013; revised March 17, 2014.

The authors are with Computer Systems Department of Mehran University, Jamshoro, Pakistan (e-mail: moazzam.jawaid@faculty.muet.edu.pk).

\section{B. Infrastructure-Less (Adhoc) Networks}

Adhoc networks represent a new pattern of wireless communication for mobile nodes [1]. In adhoc networks, there is no concept of preset base stations or access point responsible for centralized path management; instead all mobile devices within each other's communication range correspond with each other directly via wireless links as shown in Fig. 1. Those located outside range; depend on other nodes to forward pass on messages as routers. The movement of nodes changes the topology frequently arising the communication problem especially as route changes time and again [2]. Since In adhoc networks every node is mobile and can be contacted dynamically by anyone, therefore every node has to behave as router working for finding and upholding routes for other nodes in the network. Adhoc networks can be very helpful in disaster search and save operations, meeting or conventions where users intend to spread information rapidly, and data acquisition operations in unfriendly terrain.

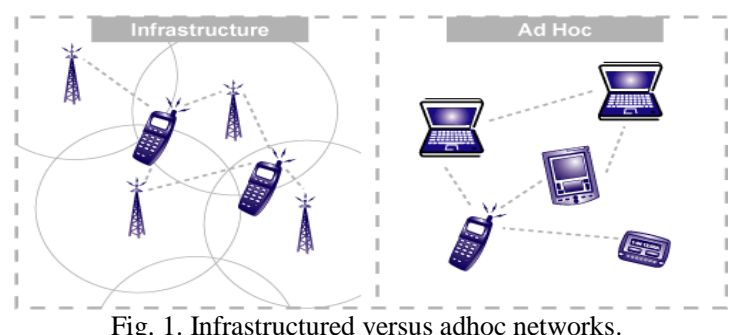

\section{Challenges in Adhoc Networks}

In recent era, adhoc networks have drawn the attention of researchers [2] as most popular fields of study and different aspects of adhoc networks have been explored in detail. But still no ultimate solution has been agreed upon. On the contrary, more questions are being raised than answered. The major open problems being discussed includes medium access control, routing of data, quality of service \& Energy Efficiency in devices. Routing [3] in adhoc networks is nontrivial because of highly dynamic nature of the devices [3], [4]. Since topology changes very frequently and every node has to provide multi hop behavior [4]. Theoretically, proposed routing protocols for adhoc networks are divided into two categories including table driven [5] (pro-active) \& on-demand (re-active) approach as shown in Fig. 2.

In pro-active routing protocols every node maintains an updated chart of routing information from all the nodes it knows. This data is updated after a specific period of time while in reactive protocols a path is formed only upon request. Research is being carried out to find a balance between these two mechanisms. For optimal routing decisions, a constant update is required regarding routes, 
delay, bandwidth but this constant update will increase the control overhead. On the other hand by using reactive approach to reduce this control overhead node may not be well aware of all possible paths to the destination.

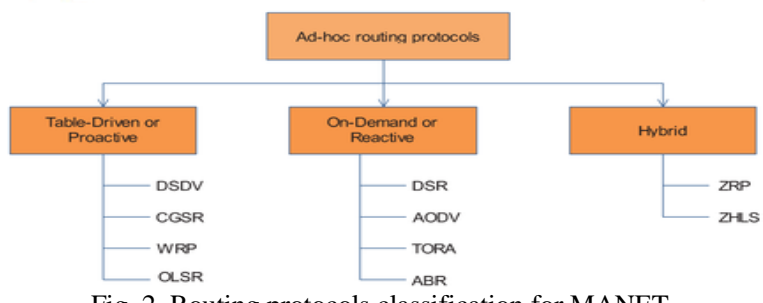

Fig. 2. Routing protocols classification for MANET.

\section{Wireless Internet}

The concept of wireless internet has also raised new research challenges. The most crucial challenge for internet protocol involves the routing of data inside wireless access networks and even among different networks that is to handle mobility of nodes [6]. The routing of data among networks, called inter-domain or macro mobility routing, has been resolved with the concept of Mobile IP protocol. However the issue of routing inside a wireless access network termed as micro mobility is still an open research topic.

\section{E. Domain Mobility}

The internet comprises of heterogeneous networks employing different technologies [6] spanned over different geographic locations and each network is termed as an administrative domain. These networks are interconnected together by using internet protocol that hides physical network differences and provides smooth global addressing. In order to avail internet service a host must be configured with valid IP addressing and this is not suitable for easy mobility of devices in wireless network. If a mobile device moves to another network, it must be configured with the setting of the new network in order to avail service. The mobility of devices can be categorized as follows.

\section{1) Macro mobility}

The movement of an internet host between different administrative domains, where a change in IP configuration is necessary for operation, is called inter domain mobility (and is also known as macro mobility).

\section{2) Micro mobility}

Micro mobility occurs within a single domain and it does not require IP addressing configuration, but it may require a change in route, and this is factor is very crucial in deciding the effectiveness of routing protocol. The impact of micro mobility on the efficiency of routing protocol can be visualized by varying the mobility parameters including speed of mobile nodes, as well as pause (static) time of nodes in the network.

\section{Simulation AND ANALYSIS}

\section{A. Platform Used}

We have used the open source platform, red hat Linux and network simulator-2 [7] for simulating the protocols of adhoc networks. NS2 is equipped with a mobility extension developed by at Carnegie Mellon University. NS2 is a detached event simulator designed for research and it supports simulating routing, TCP \& multicast protocols by allowing us to design various networks [8]. We have decided to use four prominent routing protocols including

- DSDV

- DSR

- AODV

- TORA

\section{B. Measures of Interest}

We have chosen three important parameters to analyze the impact of micro mobility including.

- Packet Delivery Ratio (PDR)

- Average End-to-End Delay of Data (EED)

- Routing Overhead(RO)

\section{1) Delivery ratio of packets}

Delivery Ratio of packets can be calculated as total packets received on the destination side divided by the total number of packets generated by sources. This ratio has been obtained by scanning trace file generated at the end of the simulation.

\section{2) Average delay}

This covers all probable delays generated in buffering during the Route discovery at intermediate nodes, or at MAC layer and propagation times. For calculating the average delay, trace file generated after simulation has been traversed and sent and received time(s) for each packet with id (li) of Trace Level (AGT) and type CBR has been marked [9].

\section{3) Routing overhead}

In order to start data transmission in the network, different routes are to be discovered and then constantly these routes are to be updated and maintained. These routes are maintained in form of routing tables and different nodes exchange their routing table information [10]. This exchange of routing information is termed as overhear or control information, as this is not the data to be transported in the network instead this is pre requisite for making the network operational. We have analyzed the routing overhead associated with those protocols by separating the packets tagged with routing symbol.

\section{TESTING ENVIRONMENT}

Specific conditions have been considered for simulation during this study, however if we change these input conditions, there may be variations in results for instance, for a tiny area (dimensions of adhoc network are relatively small), the likelihood for Packet drop is comparatively less, and if the vicinity is expanded, undoubtedly the Packet fall ratio would increase [11]. However, the routing overhead is the intrinsic property of the routing protocol [12], which shows the efficiency of the protocol.

\section{A. Traffic Model/Connection Pattern}

For running simulation in NS2, the first step is to generate the traffic model for our adhoc network [13], [14], and we have generated several models by using the available utility in /root/mz/ns/indep-utils/cmu-scen-gen/ 
folder namely cbrgen.tcl as shown in Fig. 3.

ns cbrgen tcl [-type cbr] [-nn nodes] [-seed seed] [-mc connections] [-rate rate]

\begin{tabular}{|l|l|}
\hline Parameter & Values \\
\hline Type of Traffic & CBR \\
\hline Number of Node & 60 \\
\hline Number of connection & $10,20,30,40$ \\
\hline Seed & 10 \\
\hline Rate & $8 \mathrm{Kbps}$ \\
\hline
\end{tabular}

Table 6.1 Connection pattern for simulation

ns cbrgen.1

$0.000125>\operatorname{cpl}$

Fig. 3. Traffic model /connection pattern parameters.

\section{B. Mobility Models}

After having traffic models for an adhoc network, now we need to generate the mobility model, having different values of mobility factor. We have done this by using the utility. /stdest located in /root/mz/indep-utils/cmu-scengen/setdest [13], [14] in the following manner. As mobility is measured in terms of pause time, so we have generated models by varying the pause time as shown below in Fig. 4 .

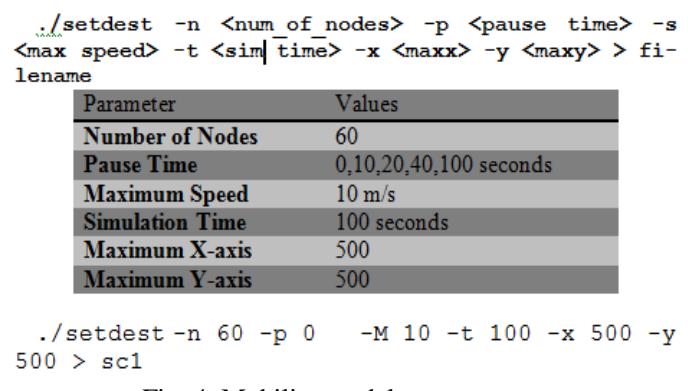

Fig. 4. Mobility model parameters.

\section{RESUltS AND CONCLUSION}

The section below Fig. 5-Fig. 7 shows the result generated after running simulations for the data sets representing different scenarios of adhoc networks. Pause time has been varied to check its impact on output parameters.

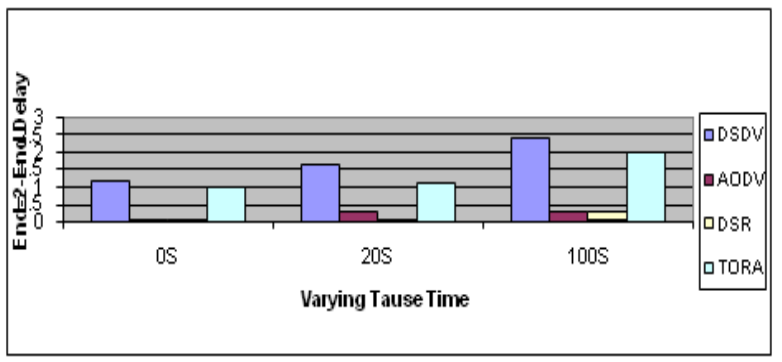

Fig. 5. (a) Average EED for 10 sources (changing paused time).

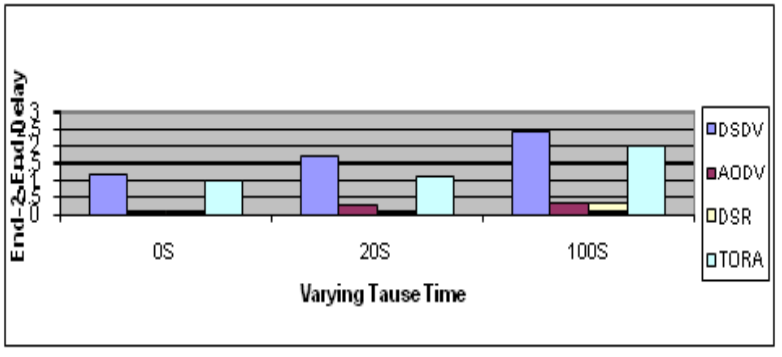

Fig. 5. (b) Average EED for 20 sources (changing paused time).

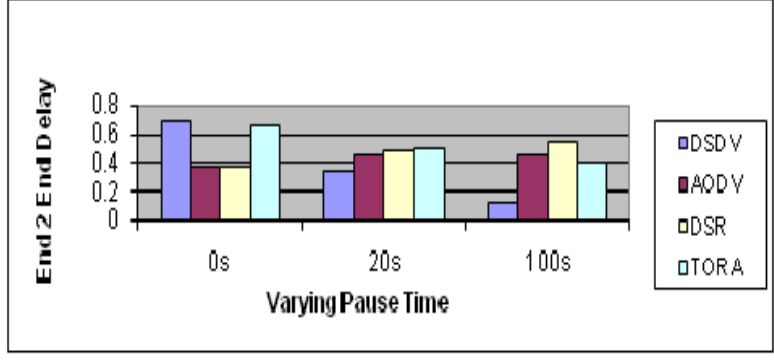

Fig. 5. (c) Average EED for 30 sources (changing paused time).

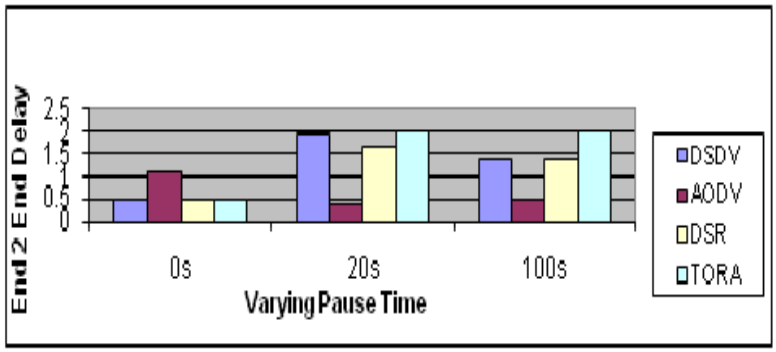

Fig. 5. (d) Average EED for 40 sources (changing paused time).

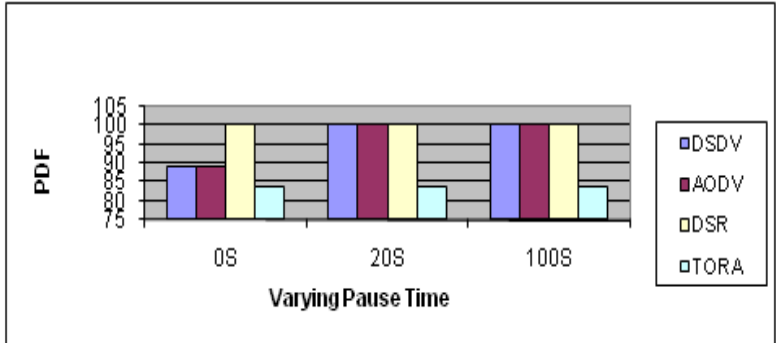

Fig. 6. (a) Packet delivery fraction for 10 (changing paused time)

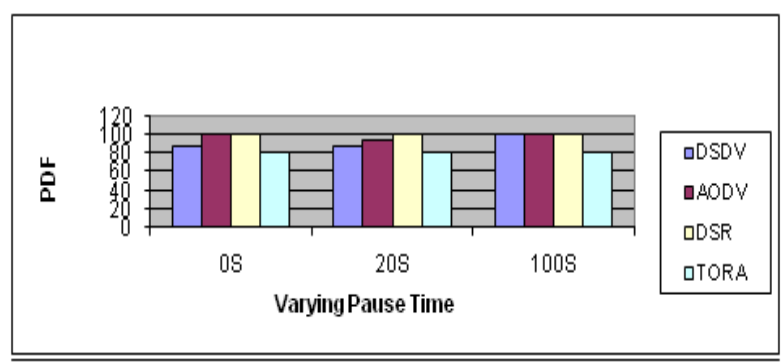

Fig. 6. (b) Packet delivery fraction for 20 sources (changing paused time)

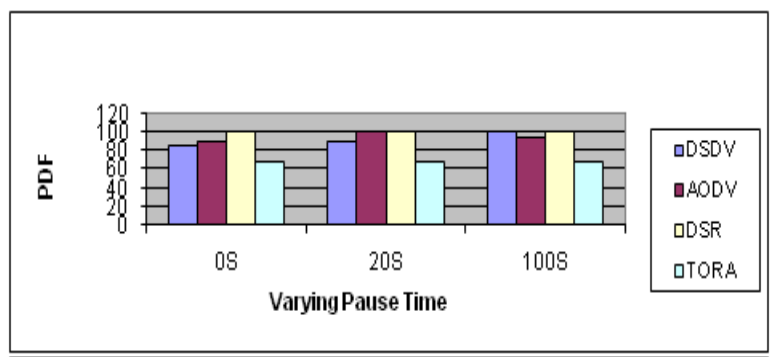

Fig. 6. (c) Packet delivery fraction for 30 sources (changing paused time).

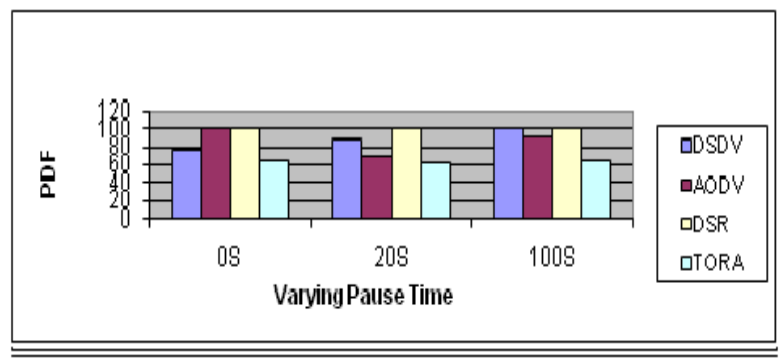

Fig. 6. (d) Packet delivery fraction for 40 sources (changing paused time) 


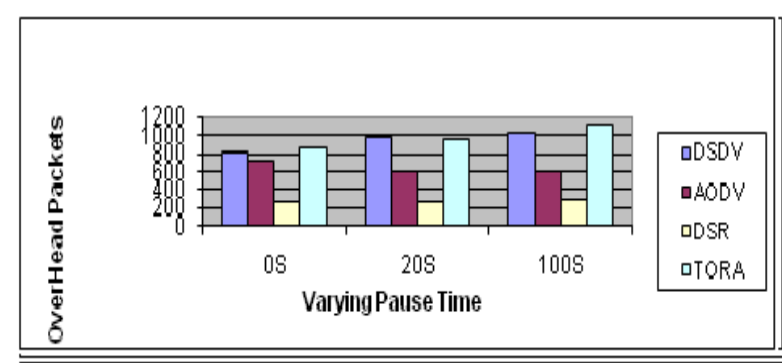

Fig. 7. (a) Control overhead for 10 nodes (changing paused time).

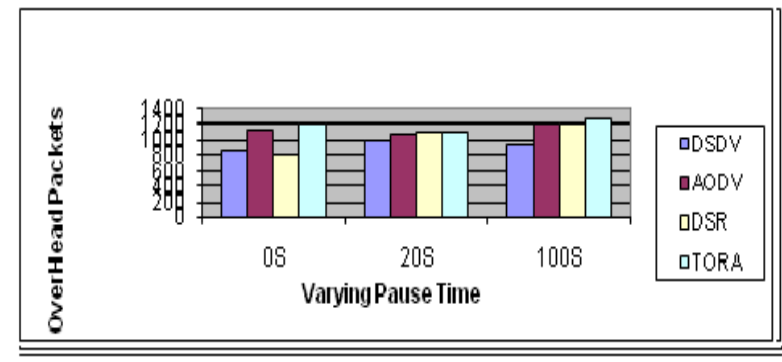

Fig. 7. (b) Control overhead for 20 nodes (changing paused time).

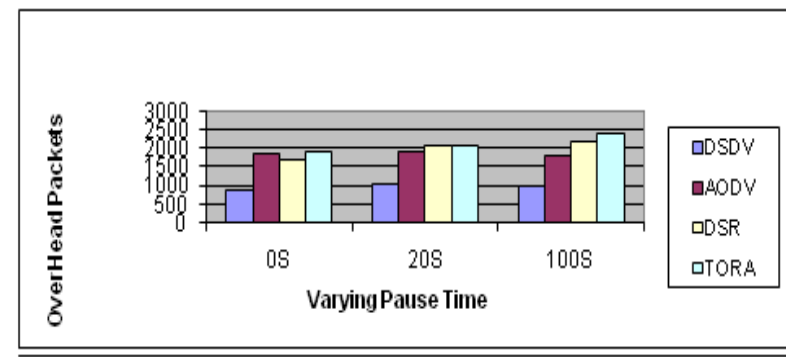

Fig. 7. (c) Control overhead for 30 nodes (changing paused time).

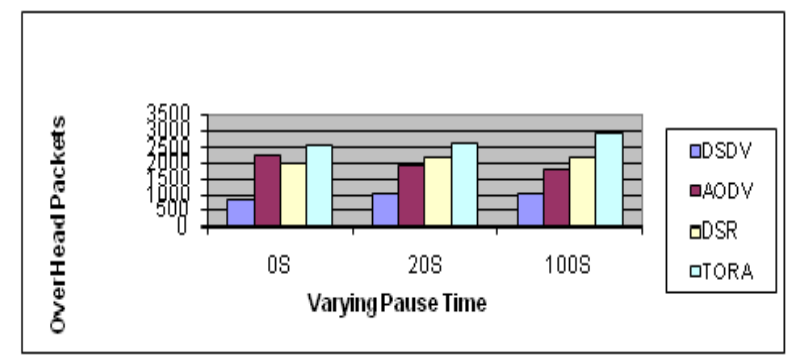

Fig. 7. (d) Control overhead for 40 nodes (changing paused time).

\section{CONCLUSION}

After analyzing the effects of Micro mobility in mobile ad-hoc networks it can be concluded that routing protocols are extremely affected by mobility and an exceptional routing protocol is needed for a dense network of highly mobile nodes. However, analysis of results shows that AODV and DSR have overall good performance at high mobility. Since DSR is based on source routing and overhead of packets is increased when size of network increases, AODV seems more desirable and stands moderate, because it offers tolerable end to end delay, standard routing overhead and also fine packet delivery fraction. In future this work can be extended in context of incorporating security mechanism in AODV.

\section{ACKNOWLEDGEMENT}

We must acknowledge the efforts made by our student, Engr. Abdul Jabbar Jamali who has participated in this research actively.

\section{REFERENCES}

[1] L. Zhou and Z. J. Haas, "Securing adhoc networks," Department of Computer Science, School of Electrical Engineering, Cornell University, Ithaca, NY.

[2] W. Li and A. Joshi. Security issues in mobile adhoc networks-a survey. [Online]. Available: http://www.csee.umbc.edu/ wenjia1/699_report.pdf.

[3] A. Yadav and P. Joshi. Performance of flat routing protocols in MANET. International Journal of Electronics and Computer Science Engineering. [Online]. 1(4). pp. 2035-2041. Available: http://www.ijecse.org/wp-content/uploads/2012/09/Volume1Number-4PP-2035-2041.pdf.

[4] G. Singh and J. Singh, "MANET: issues and behavior analysis of routing protocols," International Journal of Advanced research in Computer Science and Software Engineering, vol. 2, issue 4, pp. 219227, April 2012

[5] N. S. Yadav and R. P. Yadav. Performance comparison and analysis of table- driven and on-demand routing protocols for mobile ad-hoc networks. [Online]. Available: http://citeseerx.ist.psu.edu/viewdoc/download? doi= 10.1.1.110.13\& rep=rep1\&type=pdf.

[6] P. Zhang and B. L, "A survey of internet mobility," presented at the International Conference on Network-Based Information Systems, NBIS '09, Aug. 19-21, 2009.

[7] R. Kong, "The simulation for network mobility based on NS2," in Proc. the International Conference on Computer Science and Software Engineering, Dec. 12-14, 2008, pp. 1070-1074.

[8] S. Siraj, A. K. Gupta, and R. Badgujar, "Network simulation tools survey," International Journal of Advanced Research in Computer and Communication Engineering, vol. 1, issue 4, pp. 201-210, June 2012.

[9] J. G. Jayanthi, S. A. Rabara, and M. Arokiaraj, "IPv6 MANET: an essential technology for future pervasive computing," in Proc. the Second International Conference on Communication Software and Networks, Feb. 26-28, 2010, pp. 466, 470,

[10] Mobile adhoc networks challanges, applications and their routing protocols. [Online]. Available: http://gnindia.dronacharya.info/download/Research/DEC_Research_J ournal_Book_1905 2011.pdf

[11] B. Arkko, J. Eggert, and L. Rajahalme, "A node identity internetworking architecture," in Proc. 25th IEEE International Conference on Computer Communications, April 2006, vol. 1, no. 6 , pp. 23-29.

[12] S. Michaelis, A. Lewandowski, K. Daniel, and C. Wietfeld, "Macro mobility prediction for high-priority resource reservation in wireless networks," in Proc. the IEEE International Symposium on ISWCS '08, Oct. 21-24, 2008, p.184.

[13] Y. Chaba, Y. Singh, and M. Joon, "Simulation based performance analysis of on-demand routing protocols in MANETs," in Proc. the Second International Conference on Computer Modeling and Simulation, Jan. 22-24, 2010, pp. 80-83.

[14] Amutha and S. Balasubramanian, "Secure implementation of routing protocols for wireless Ad hoc networks," in Proc. the International Conference on Information Communication and Embedded Systems, Feb. 21-22, 2013, pp. 960-965.

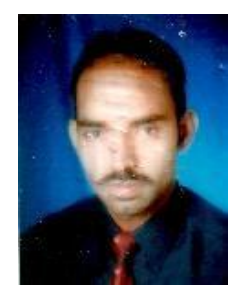

Moazzam Jawaid is working as an assistan professor in the Department of Computer Systems Engineering in Mehran University of Engg. \& Technology, Jamshoro, Pakistan. He has got his M.E in communication systems \& networks. Currently he is perusing his $\mathrm{PhD}$. Mr. Moazzam is a member of several professional organization including IACIST PEC and IJENS. He is a reviewer of several journals internationally. 